\title{
Bacteriological profile by blood culture among acute lymphoblastic leukemic children hospitalized with neutropenia and fever in a tertiary level hospital, Bangladesh.
}

\author{
Farook Ahamed ${ }^{1}$, Shahin Ara Begum ${ }^{1}$, Mohammad Jobayer $^{1}$, Zeenat Afroz ${ }^{1}$, Mizanur Rahman ${ }^{1}$, Sazzad Bin Shahid ${ }^{1}$, \\ Kamrunnesa ${ }^{2}$, Afiqul Islam ${ }^{3}$. \\ ${ }^{1}$ Department of Microbiology, Dhaka Medical College, Dhaka. ${ }^{2}$ Department of Pharmacology, Shahabuddin Medical \\ College, Dhaka. ${ }^{3}$ Department of Paediatric Hematology and Oncology, Bangabandhu Sheikh Mujib Medical University \\ Dhaka, Bangladesh.
}

Submitted on: 20 July, 2014. Accepted on: 10 October, 2014

\begin{abstract}
:
Bacterial infection is an important complication among children with cancer, who are under chemotherapy. Bacterimea may occur during chemotherapy, which is an invasive procedure and also due to reduced number of neutrophil count. The study was conducted in a tertiary level hospital, Bangabandhu Sheikh Mujib Medical University in Bangladesh from March to September 2010. The aim of this study was isolation and identification of bacteria from blood culture among children with febrile neutropenia who were under chemotherapy. Blood samples were collected from 30 febrile neutropenic children and culture was done by traditional culture method using Trypticase soy broth for primary culture. Out of 30 blood samples, culture were positive in $6(20 \%)$ and were negative in $24(80 \%)$ samples. Two third of the culture positive patients had absolute neutrophil count (ANC) less than 100 cells $/ \mathrm{cmm}$. The isolated organisms were Pseudomonas spp (33.3\%) Staphylococcus aureus (33.3\%), Esch. coli (16.7\%) and Acinetobacter spp (16.7\%). Amikacin, imipenem and meropenem were the most effective antibiotics having $100 \%$ sensitivity. Blood culture is essential to evaluate the febrile neutropenic children who were under chemotherapy.
\end{abstract}

Key word: Antibiotics, Bangladesh, Blood culture, Febrile neutropenia.

\section{Introduction:}

Major advances have been achieved in the management of patients with cancers, such that patients with previously untreatable illness may now receive therapy and survive ${ }^{1}$. Bacterial infections are common and potentially serious complications of cancer treatment ${ }^{2}$. With aggressive use of chemotherapy and antimicrobial therapy that has necessitated use of more invasive procedures, may contribute to increased susceptibility to infections. The net result is that patients surviving severe underlying diseases may succumb to bacterial, fungal, viral or protozoan infections ${ }^{3}$. Fever during chemotherapy induced neutropenia can be the first sign of bacterial infection and therefore requires prompt and careful attention. In the early 1970s, the introduction of empirical use

\section{Correspondence:}

Dr. Farook Ahamed

Lecturer

Department of Microbiology

Dhaka Medical College, Dhaka-1000, Bangladesh.

Tel: +8802-55165081, Mobile: 01715-005842

Email: dr.farook25@gmail.com of intravenous broad-spectrum antibiotics for febrile neutropenic patients reduced infection-related mortality significantly ${ }^{2}$. Oral temperature $\geq 38.0^{\circ} \mathrm{C}(100.40 \mathrm{~F})$ for duration $\geq 1$ hour indicates a febrile state 4 . The most important risk factor for infection is an absolute neutrophil count (ANC) less than 500 cells/cumm and its duration ${ }^{2}$.

The classic beta-lactam and/or an aminoglycoside combination has long been considered the best therapeutic approach for febrile neutropenia because of its broader spectrum, potential synergistic activity against Gram negative rods, and its potential ability to reduce the emergence of resistant strains $s^{4,5}$. With time the resistance to beta-lactam antibiotics has been on the rise and warrants close monitoring of the sensitivity patterns. Therefore, new treatment strategies for febrile neutropenic patients for bacterial infection are under investigation. Though the exact statistics of incidence and prevalence of febrile neutropenia among the patients with cancer in our country is not available but it is the common opinion that the number of such kind of problem is increasing day by day. The aim of this study was to isolate and identify bacteria by blood culture during episodes of fever and neutropenia in children with cancer. 


\section{Materials and Methods:}

It was a cross sectional prospective study. The study was conducted in the Department of Pediatric Haematology and Oncology, in Bangabandhu Sheikh Mujib Medical University (BSMMU), Dhaka, Bangladesh for a period of 6 months from March 2010 to September 2010. All microbiological works were done in the Department of Microbiology and Immunology, BSMMU.

Study population: Children suffering from malignancies and undergoing therapy admitted in the Department of Pediatric Haematology and Oncology, BSMMU. Children between 1-15 years of age of both sexes with bone marrow or biopsy evidence of malignancy and clinical features of febrile neutropenia were included. The exclusion criteria were any patients on antibiotic treatment and very ill children needed ICU support.

\section{Sample size:}

The sample size was determined by the following formula

Here $\mathrm{p}=0.07$, Isais-Agdeppat $\mathrm{d}^{2}$ and Bravo determined the bacterial blood isolates in pediatric cancer patients and the prevalence rate was $7 \%{ }^{6}$. According to this formula the estimated sample size was 30 .

\section{Data collection technique:}

Required data was collected by face to face interview. The diagnosis of febrile neutropenia $(\mathrm{FN})$ was made as absolute neutrophil count (ANC) of $<500 / \mathrm{cmm}$ or a count of $<1000 / \mathrm{cmm}$ with a predicted decline to $500 / \mathrm{cmm}$, associated with single oral temperature of $238.3^{\circ} \mathrm{C}$ or a temperature of $\geq 38.0^{\circ} \mathrm{C}$ for $\geq 1$ hour $^{6}$. All participants were subjected to be thorough clinical examination and tests for complete blood count, peripheral blood film, blood culture and sensitivity, urine culture and sensitivity and CRP estimation. Antibiotic was started after collection of the specimen.

\section{Sample collection:}

With all aseptic precaution $2.5 \mathrm{ml}$ blood were collected from antecubital vein from 30 febrile neutropenic children with cancer. Blood was collected before starting antibiotics.

\section{Blood culture:}

Blood culture was done by Traditional method. Trypticas soy broth was used for primary culture. Blood agar, chocolate agar and MacConkey agar media were used for subculture. In case of positive blood culture, bacterial identification was done by colony morphology, Gram staining and different biochemical tests as per standard guidelines.

\section{Antimicrobial susceptibility testing:}

Antimicrobial susceptibility pattern of the isolated organisms were determined by Kirby-Bauer disc diffusion method ${ }^{7}$ using commercially available antibiotic discs (Oxoid, UK). The organisms were tested against different antibiotics and commonly used discs were amikacin, ceftazidime, ceftriaxone, ciprofloxacin, gentamicin, imipenem, meropenem, Co-trimoxazole, netilmicin. Zone of inhibition was recorded as 'Sensitive' or 'Resistant' according to the CLSI guideline ${ }^{8}$.

\section{Ethical consideration:}

The research protocol was approved by Ethical committee of Bangladesh College of Physician and Surgeons (BCPS), Dhaka. An informed written consent was taken from the legal guardians of the patients. They were informed about the purpose of this study and it was also made clear to them that who did not want to participate in the study should feel free to withdraw. The anonymity of the participants and confidentially of information was maintained.

\section{Statistical analysis of data:}

Statistical analyses of the results were obtained by using window based computer software devised with Statistical Packages for Social Sciences (SPSS-15) (SPSS Inc, Chicago, USA).

\section{Results:}

Children between 1-15 years of age with bone marrow or biopsy evidence of malignancy and clinical features of febrile neutropenia were enrolled in the study. Among the 30 respondents $6(20 \%)$ were positive for blood culture.Mean \pm SD age was $5.47 \pm 3.25$ years and range of age was 2-14 years. Two third $(66.7 \%)$ of the respondents were in the age group of $\leq 5$ years (Fig-1). Among the respondents 19 (63.3\%) were male and $11(36.7 \%)$ were female. Male to female ratio was 1: 0.58 (Fig-2).

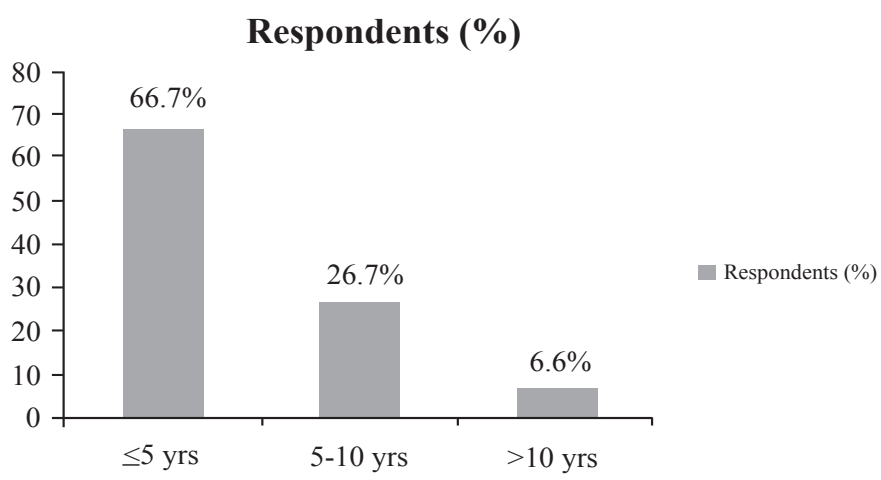

Figure 1: Distribution of age of the respondents. 


\section{Distribution of sex of the respondents}

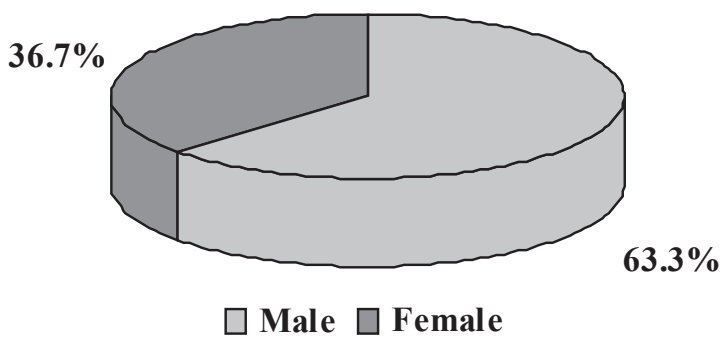

Figure2: Distribution of sex of the respondents.

Among the 6 patients with positive blood culture $2(33.3 \%)$ patients had absolute neutrophil count (ANC) more than 100 cells/cmm and $4(66.7 \%)$ patients had ANC less than 100 cells/cmm (Table-I).From the positive blood culture cases among the 6 isolated organisms Pseudomonas spp were (33.3\%) and Staphylococcus aureus were (33.3\%) followed by Esch. coli (16.7\%) and Acinetobacter spp (16.7\%) (Table-2).

Table 1: Distribution of ANC among the blood culture positive respondents $(\mathrm{N}=6)$.

\begin{tabular}{lcc}
\hline ANC & Frequency & Percentage (\%) \\
\hline$>100$ cells $/ \mathrm{cmm}$ & 2 & 33.6 \\
$<100$ cells $/ \mathrm{cmm}$ & 4 & 66.4 \\
Total & 6 & 100.0 \\
\hline
\end{tabular}

Table 2: Organisms isolated from blood culture of the respondents $(\mathrm{N}=6)$.

\begin{tabular}{lcc}
\hline Organism in blood culture & Frequency & Percentage (\%) \\
\hline Pseudomonas spp. & 2 & 33.3 \\
Staphylococcus aureus & 2 & 33.3 \\
Esch. coli & 1 & 16.7 \\
Acinetobacter spp. & 1 & 16.7 \\
\hline
\end{tabular}

Table-3 showing the sensitivity pattern of the isolated organisms from blood culture. The most effective antibiotics were amikacin, imipenem, meropenem, netilmicin whereas ciprofloxacin, ceftriaxone and Co-trimoxazole were the most resistant antibiotics.

Table 3: Antimicrobial susceptibility pattern of isolated bacteria $(\mathrm{N}=6)$.

\begin{tabular}{lcccccccccccc}
\hline Isolated & \multicolumn{10}{c}{ Sensitivity (\%) } \\
\cline { 2 - 12 } organism & Ak & Cro & Caz & G & Cip & Mem & Imp & Net & Cot & Va & Lzd \\
\hline Pseudomonas & 100 & 0 & 100 & 100 & 50 & 100 & 100 & 100 & 0 & - & - \\
S.aureus & 100 & 100 & 100 & 100 & 50 & 100 & 100 & 100 & 50 & 100 & 100 \\
Esch. coli & 100 & 0 & 0 & 0 & 00 & 100 & 100 & 100 & 0 & - & - \\
Acinetobacter & 100 & 0 & 0 & 0 & 0 & 100 & 100 & 0 & 0 & - & - \\
\hline
\end{tabular}

Ak-amikacin, Cro-ceftriaxone, Caz-ceftazidime, G-gentamicin, Cip-ciprofloxacin, Imp-imipenem, Mem-meropenem, Cot-Co-trimoxazole, Net-netilmicin, Va-vancomycin, Lzd-linezolid.

\section{Discussion:}

The last few decades have seen tremendous improvements in survival of children diagnosed with cancer, with the 5-year survival rate approaching $80 \%{ }^{9}$. Febrile neutropenia is a common clinical problem among pediatric cancer patients ${ }^{10}$. This often necessitates hospitalization and immediate empiric broad spectrum antimicrobial therapy ${ }^{6}$. Leukaemia is the commonest cancer in children (0-14 years), representing a third of all malignancies. Incidence rates increase to a peak around age 3-4 years and then decline ${ }^{11}$.

In the present study out of 30 respondents with leukaemia two third were in the age group of $\leq 5$ years. Acute lymphoblastic leukemia (ALL) typically develops in children between one and ten years of age, although it can occur at any age 12. The result of this study coincide with the finding of Esparza and Sakamoto13 who reported that the peak incidence of childhood ALL occurs between the age of 3 and 4 years. Leukemia is the most common malignancy of childhood, and acute lymphoblastic leukemia is the most common type of leukemia in children and more common in males ${ }^{12}$. In the present study among the respondents $63.3 \%$ were male. Esparza and Sakamoto and Stabell et al also found that ALL is slightly more common in boys than in girls ${ }^{10,13}$. Twenty percent of the leukaemic patients with clinical features of neutropenia were found positive in blood culture. The positivity rate matched with study by Rackoff et al and Stabell et al both of whom found the rate to be $21 \% \%^{10,14}$. Isais-Agdeppa and $\mathrm{Bravo}^{6}$ in the Philippines in a retrospective study found only $7 \%$ of the patients had positive growth in the blood culture among the pediatric cancer which is much lower than the present study. The discrepancy of the culture positivity of different studies may be due to the varying prevalence of septicemia from country to country and even area to area. Developing countries have insufficient infection control measures, limited financial or administrative support which may be responsible for higher rate of infection ${ }^{15}$. Among 6 isolated organisms $80 \%$ were gram negative bacteria and the isolated bacteria were Pseudomonas spp, Esch coli, Acinetobacter spp and Staph aureus. Hana et a ${ }^{16}$ reported that in children with cancer infection Viridans Streptococci, Pseudomonas spp. and Esch coli were the most frequently isolated organisms. Tezcan et al ${ }^{17}$ in their study among children with neutropenia and cancer Staphylococci and Escherichia coli were the most commonly isolated organisms.

The absolute neutrophil count among all the 30 respondents were less than 500 cells $/ \mathrm{cmm}$ and two third of the patients with positive blood culture had ANC less than 100 cells $/ \mathrm{cmm}$. Patients with counts of $<100$ cells $/ \mathrm{cmm}$ are at greater risk than 
are those with counts of $<500$ cells $/ \mathrm{cmm}^{4}$. Jones et al ${ }^{18}$ in a study found that certain high risk infections were more common with an ANC $<200 \mathrm{cell} / \mathrm{cmm}$. Antibiotic sensitivity pattern of the isolated organisms shows that the most effective antibiotics were amikacin, meropenem, imipenem, netilmicin.

\section{Acknowledgement:}

We acknowledge the Department of Pediatric Haematology and Oncology, Bangabandhu Sheikh Mujib Medical University, Dhaka, Bangladesh for providing the sample collection facility.

\section{Conflict of interest:}

We do not have any potential conflicts of interest.

\section{References:}

1. Khan MA, Siddiqui BK, Shamim A, et al. Emerging bacterial resistance patterns in febrile neutropenic patients: experience at a tertiary care hospital in Pakistan. JPMA 2004; 54: 357-359.

2. Nijhuis CO, Kamps WA, Daenen SMG, et al. Feasibility of withholding antibiotics in selected febrile neutropenic cancer patients. J Clin Oncol 2005; 23(30): 7437-7444.

3. Viscoli $\mathrm{C}$ and Castagnola E. Treatment of febrile neutropenia: what is new? Curr Opin Infect Dis 2002; 15: 377-382.

4. Hughes WT, Armstrong D, Bodey GP, et al. Guidelines for the use of antimicrobial agents in neutropenic patients with cancer. CID 2002; 34: 730-751.

5. Viscoli C. The evolution of the empirical management of fever and neutropenia in cancer patients. J Antimicrob Chemother 1998; 41: 65-80.

6. Isais-Agdeppa AT and Bravo L. A five-year retrospective study on the common microbial isolates and sensitivity pattern on blood culture of pediatric cancer patients admitted at the Philippine general hospital for febrile neutropenia. PIDSP J 2005; 9(2): 19-26.

7. Bauer AW, Kirby WMM, Sherris JC, Turck M. Antibiotic susceptibility testing by a standardized single disk method. Am J Clin Pathol 1966; 36: 493-6.
8. CLSI. Performance standards for antimicrobial susceptibility testing; Informational supplement, CLSI document, Wayne, PA: Clinical and Laboratory Standards Institute, 2010.

9. Bhatia S. Cancer Survivorship-Pediatric Issues. Hematol 2005; $1: 507-515$.

10.Stabell N, Nordal E, Wiger K, et al. Febrile neutropenia in children with cancer; a retrospective Norwegian multicentre study. Presentation: 17th European Congress of Clinical Microbiology and Infectious Diseases ICC, Munich, Germany, 2007.

11. Shah A and Coleman MP. Increasing incidence of childhood leukaemia: a controversy re-examined. $\mathrm{Br} \mathrm{J}$ Cancer 2007; 97(7): 1009-1012.

12. Robison LL. General principles of the epidemiology of childhood cancer. In: Pizzo PA, Poplack DG, eds. Principles and practice of pediatric oncology. 3d ed. Philadelphia, USA: Lippincott-Raven, 1997: pp:1-10.

13.Esparza SD and Sakamoto KM. Topics in pediatric leukemia- Acute lymphoblastic leukemia. Med Gen Med 2005; 7(1): 23.

14.Rackoff WR, Gonin R, Robinson C, Kreissman SG, Breitfeld PB. Predicting the risk of bacteremia in childen with fever and neutropenia. J Clin Oncol 1996; 14: 919-924.

15. Bicudo D, Batista R, Furtado GH, Sola A, De Medeiros EAS. Risk factors for catheter-related bloodstream infection: a prospective multicenter study in Brazilian intensive care units. Braz J Infect Dis 2011; 15(4): 328-331.

16.Hana H, Patricia MF, Katherine MK, Deo Kumar S, Aditya HG. Etiology and clinical course of febrile neutropenia in children with cancer. J Pediatr Hematol Oncol 2009; 31(9): 623-629.

17. Tezcan G, Kupesiz A, Ozturk F, et al. Episodes of fever and neutropenia in children with cancer in a tertiary care medical center in Turkey. Pediatr Hematol Oncol 2006; 23(3): 217-229.

18. Jones GR, Konsler GK, Rose P, Dunaway RP, Pusek SN. Infection risk factors in febrile, neutropenic children and adolescents. Pediatr Hematol Oncol 1996; 13(3): 217-229. 